\title{
Références bibliographiques du dossier " programmes et politiques éducatives »
}

Maryannick Vervisch-Lestrade

\section{OpenEdition}

1 Journals

Édition électronique

URL : http://journals.openedition.org/ries/3442

DOI : 10.4000/ries.3442

ISSN : 2261-4265

Éditeur

Centre international d'études pédagogiques

\section{Édition imprimée}

Date de publication : 1 décembre 1996

Pagination : 135-142

ISSN : 1254-4590

\section{Référence électronique}

Maryannick Vervisch-Lestrade, «Références bibliographiques du dossier « programmes et politiques éducatives » », Revue internationale d'éducation de Sèvres [En ligne], 12। 1996, mis en ligne le 28 août 2013, consulté le 10 décembre 2020. URL : http://journals.openedition.org/ries/3442 ; DOI : https:// doi.org/10.4000/ries.3442 


\title{
Références bibliographiques
}

\author{
Maryannick Vervisch-Lestrade
}

Cette bibliographie thématique présente une sélection de documents récents consultables au CIEP. Réalisée à partir de références françaises et étrangères, elle est organisée, après une présentation de documents généraux, en fonction de trois thèmes essentiels relatifs aux programmes d'enseignement: objectifs et politiques; contenus; acteurs.

\section{Données générales}

CONSEIL DE L'EUROPE, Atelier de recherche sur les programmes d'enseignement secondaire, La Valette (Malte) 6-9 octobre 1992, (DECS/Rech 92). Les interventions des participants, venus de nombreux pays européens, ont abordé le sujet sous différents angles: objectifs des réformes récentes; critères de choix et structuration des contenus; prise en compte des valeurs, des aspects globaux et des nouvelles connaissances; influence de l'économie et du monde du travail; développement personnel de l'élève et préparation à sa vie future.

DEMONQUE Chantal coord., Qu'est-ce qu'un programme d'enseignement?, Paris, Centre national de documentation pédagogique, Hachette éducation, 1994, 172 p., (Ressources formation. Enjeux du système éducatif).

L'ouvrage explique comment, par qui et selon quels objectifs sont élaborés les programmes. Il précise aussi leurs liens avec les savoirs de référence qui les inspirent. A travers des chapitres concernant différentes matières d'enseignement, les auteurs abordent ces sujets sous des angles disciplinaires, mais aussi pédagogiques, institutionnels, historiques ou comparatifs.

EURYDICE, Réseau d'information sur l'éducation dans l'Union européenne et les pays de l'AELE-EEE, L'enseignement préscolaire et primaire dans I'Union européenne, Bruxelles, Eurydice, 1994, 119 p.

Dans ce document qui contient des données de base sur l'enseignement des enfants de 2 à 12 ans dans les États membres, la seconde partie concerne l'élaboration et le contenu des programmes et l'évaluation des élèves. De nombreux tableaux, graphiques et cartes illustrent le texte.

ORGANISATION DE COOPÉRATION ET DE DÉVELOPPEMENT ÉCONOMIQUES, Redéfinir le curriculum: un enseignement pour le $x x l^{e}$ siècle, Paris, OCDE, 1994, 274 p., (Documents OCDE).

Le rapport donne l'état des réflexions et des actions consacrées aux programmes scolaires et menées par le Centre pour la recherche et l'innovation dans l'enseignement au cours de la dernière décennie. A partir de ces travaux, le livre traite des perspectives et des politiques futures. L'engagement d'un enseignement de qualité pour tous s'insère dans l'interaction entre les initiatives des écoles, les priorités nationales et les préoccupations sociales et éducatives.

WESTON Penelope, BARRETT Elisabeth, The Quest for Coherence: Managing the Whole Curriculum 5-16, Slough (Royaume-Uni), National Foundation for Educational Research, 1992, II-241 p.

Destiné aux praticiens, ce livre fait le point sur le nouveau curriculum britannique. Changements dans les programmes d'enseignement dans l'enseignement primaire et secondaire depuis 1988, struc- 
tures de gestion et mise en œuvre des programmes, signification et faisabilité de ceux-ci, tels sont les différents thèmes abordés.

Des informations sur le sujet figurent également dans des ouvrages plus généraux tels que:

CHAMPY Philippe dir., ETEVE Christiane dir., Dictionnaire encyclopédique de l'éducation et de la formation, Paris, Nathan, 1994. Articles "Curriculum", p. 218-223, et "Programmes scolaires", p. 798-802.

HUSEN Torsten ed., POSTLETHWAITE T. Neville ed., International Encyclopedia of Education, $2^{\text {nd }}$ ed., Oxford, Elsevier Sciences, 1994, Vol. 3: plusieurs articles abordent différents aspects du "curriculum».

Les revues spécialisées publient également des articles sur ce thème; on peut citer:

- Curriculum Studies, publié 3 fois par an par Triangle Journals (PO Box 65, Wallingford, Oxfordshire OX10 OYG, Royaume-Uni);

- Educational Review, publié 3 fois par an par Carfax publishing company (PO Box 25, Abingdon, Oxfordshire OX14 3UE, Royaume-Uni);

- Studies in Educational Evaluation, publié 4 fois par an par Pergamon, Elsevier Sciences, (The boulevard, Langford lane, Kidlington, Oxford OX 1GB, Royaume-Uni).

En France, le Bulletin officiel de l'Éducation nationale, hebdomadaire, publie les textes réglementaires concernant les programmes, ainsi, dernièrement, la "Consultation nationale sur les projets de programmes de langues vivantes 2 pour les classes de quatrième et troisième", dans le Bulletin officiel du 26 sept. 1996, n 5 , p. 1-172. Ces textes sont ensuite regroupés dans les chapitres du tome $\mathrm{V}$ du Recueil des lois et règlements et également publiés, par niveau et discipline, dans la collection "Horaires, programmes, instructions ", éditée par le Centre national de documentation pédagogique.

Enfin, nous signalons un document que le ministère de l'Éducation nationale vient de faire publier:

FRANCE. Direction des Lycées et des Collèges, Accompagnement des programmes de $6^{e}$, Paris, Centre national de documentation pédagogique, 1996, 2 livrets, (collège).

L'intérêt de ce document réside dans son caractère pédagogique à destination des enseignants et dans les développements qu'il apporte en complément des instructions officielles.

\section{Quels objectifs et quelles politiques?}

AEDO-RICHMOND Ruth, RICHMOND Mark, "Recent Curriculum Change in Post-Pinochet Chile", Compare, a Journal of Comparative Education, juin 1996, vol. 26, n², p. 197-215.

L'article explique les raisons et modalités des changements intervenus dans les programmes de l'enseignement primaire depuis mars 1990. Les modifications du curriculum réalisées sous le régime de Pinochet ont entraîné ensuite des réactions critiques. Les priorités éducatives du gouvernement Aylwin s'inscrivent dans un contexte socio-politique et économique spécifique, cependant qu'émerge une décentralisation de l'enseignement. 
BARTLETT Leo, "National Curriculum in Australia: an Instrument of Corporate Federalism", British Journal of Educational Studies, août 1992, vol. XXXX, $n^{\circ} 3$, p. $218-238$.

Les relations entre élaboration du curriculum national et structures administrative et politique sont le sujet de cet article selon lequel le curriculum serait un instrument aux mains du pouvoir fédéral représenté par l'AEC (Australian Education Council). Le rappel historique de son élaboration durant les années 70 et 80 , dans des circonstances économiques et politiques différentes, précède la description de l'idéologie qui l'inspire. Aujourd'hui, les valeurs et principes des concepteurs du système sont: "néo-corporatisme», rationalisme économique, stratégie managériale et capital humain. La démarche adoptée exclut de la préparation des programmes les praticiens de l'éducation: enseignants, parents, formateurs, étudiants.

CHITTY Clyde ed., "International Perspectives on the Curriculum: Special Issue", Educational Review, juin 1993, vol. 45, n², p.107-173.

Les différents articles de ce numéro spécial apportent des points de vue variés sur la question des programmes. La mise en œuvre du curriculum national britannique est abordée à travers ses relations avec la politique et l'idéologie et dans la perspective européenne. D'autres auteurs présentent les spécificités des curricula aux États-Unis, en Australie, Nouvelle-Zélande et au Kenya.

COULBY David, "Ethnocentricity, Post Modernity and European Curricular Systems", European Journal of Teacher Education, oct. 1995, vol. 18, $n^{\circ} 2-3, p .143-153$.

Centré sur les théories sociales et culturelles de la modernité et de la post-modernité, l'article examine les conséquences, sur la pédagogie et les programmes, de la naissance d'une supranationalité européenne.

DEFARD Sylvie, La dimension européenne à l'école: rapport de stage, Cardiff, 20-26 octobre 1993, Reims, MAFPEN, oct. 1993, 14 p.

Dans ce compte rendu du colloque intitulé «la dimension européenne dans les programmes scolaires", l'auteur, après avoir mis l'accent sur l'intérêt d'une définition commune du terme, aborde le rôle des échelons européen, national, régional dans une politique des échanges. Puis, à travers le cas britannique, elle analyse les moyens mis en œuvre pour intégrer l'Europe dans les programmes scolaires.

HELLGREN Paul, "European Curriculum and Autonomy in Class", European Journal of Teacher Education, oct. 1994, vol. 17, $n^{\circ} 3, p .143-160$.

Dans le cadre du processus d'intégration européenne, la question d'un «curriculum européen" se pose. Il pourrait susciter une plus grande efficacité de l'enseignement, mais se heurte à des problèmes de réalisation. Ceux-ci sont liés surtout aux différences de culture et à la difficulté de définir une identité européenne.

LUBY Antony, "An Enterprising Approach to Democratising the Curriculum: Reflections on a Scottish Experience", The Vocational Aspect of Education, mars 1995, vol. 47, $n^{\circ} 1$, p. 21-33.

La prise en compte de la formation professionnelle dans l'éducation peut contribuer à démocratiser les programmes. L'expérience écossaise d'approche du monde de l'entreprise illustre cette théorie. 
Mc LEAN Martin, "The European Union and the Curriculum", Oxford Studies in Comparative Education, 1995, vol. 5 (2), p. 29-46.

L'auteur étudie les liens entre intégration européenne et contenu de l'éducation, montre les spécificités dans l'apprentissage, l'enseignement et l'évaluation, liées à des cultures politiques différentes, et s'interroge sur la nécessité d'un curriculum européen.

MSISKA F. G. W., "Some Practical Limits of Curriculum Vocationalization as a Remedy to School Leavers'Unemployment: Focus on Malawi", International Review of Education/Revue internationale de l'éducation, avril 1994, vol. $40, n^{\circ} 2, p .135-148$.

Selon l'article, une orientation professionnelle des programmes dans l'enseignement général, comme moyen de réduire le chômage des diplômés, relève du mythe. Il faut d'abord couvrir les besoins éducatifs fondamentaux et il est sans doute préférable de confier la formation professionnelle à des organismes spécialisés.

NEMO Philippe, Le chaos pédagogique: enquête sur l'enseignement des collèges et des lycées de la République, Paris, Albin Michel, 1993, 255 p. Les causes essentielles de cette situation, qui se manifeste par un manque de cohérence et de continuité et une inadaptation des contenus de l'enseignement, sont une profonde désorganisation et un blocage politique. Après un rappel historique, l'auteur examine les programmes actuels du collège et du lycée et les innovations «imaginées» par le Conseil national des programmes. Avec le soutien de responsables éducatifs convaincus de l'état d'urgence et d'une opinion publique enfin éclairée, l'auteur espère que sera entreprise la réforme nécessaire des contenus et des méthodes d'enseignement, en vue de la mise en place d'un enseignement secondaire de haut niveau.

NISBET John ed., Curriculum Reform: Assessment in Question/La réforme des programmes scolaires: l'évaluation en question, Paris, OCDE, 1993, 145 p.

Ce rapport fait partie de la série de publications du CERI sur La réforme de l'école et les innovations dans l'enseignement. Les exemples d'évaluations présentés ici montrent que les méthodes d'évaluation sont essentielles pour bien orienter le processus d'apprentissage et atteindre les objectifs assignés aux programmes scolaires.

NORDENBO Sven Erik, "What is Implied by a "European Curriculum?": Issues of Eurocentrism, Rationality and Education", Oxford Review of Education, mars 1995, vol. 21, $n^{\circ} 1$, p. 37-46.

Cet article étudie la possibilité de proposer une conception viable de cette notion. Les tendances à l'eurocentrisme peuvent être interprétées de trois façons différentes, liées aux conceptions habituelles du curriculum européen. Ce concept lui-même peut être conçu comme un instrument d'évaluation des curricula nationaux ou régionaux.

OBANYA Pai, "Case Studies of Curriculum Innovations in Western Africa", International Review of Education/Revue internationale de l'éducation, oct. 1995, vol. 41, $n^{\circ}$ 5, p. 315-336.

L'article passe en revue les tentatives d'innovation dans les programmes scolaires en Afrique occidentale depuis les années 60: usage des langues nationales, éducation à l'environnement, utilisation des émissions éducatives télévisées. Les objectifs fixés ont rarement été atteints, en raison de conditions socio-politiques et économiques défavorables mais aussi du manque d'implication de la population. 
PACHOCINSKI Ryszard, "Current Curriculum Changes in Poland: a National Report", Curriculum Studies, juin 1993, vol. 1, n², p. 215-232.

Le système éducatif polonais doit faire face à plusieurs défis importants, dont la nécessité d'élaborer un curriculum en lien avec les changements du système socio-politique et économique. L'article, après avoir présenté le système actuel, le cadre légal des réformes et les changements dans les programmes d'enseignement, discute la façon d'élaborer et de mettre en œuvre le nouveau curriculum.

SZEBENYI Péter, "Two Models of Curriculum Development in Hungary: 19721992 ", Educational Review, nov. 1992, vol. 44, n 3, p. 285-294.

Les réformes menées durant cette période, l'une sous la "dictature douce", l'autre après la chute du communisme à la fin des années 80 , sont toutes deux caractérisées par la construction d'un curriculum national centralisé. Elles sont cependant très différentes, spécialement en ce qui concerne les objectifs politiques reflétés par le calendrier et les stratégies déployées.

\section{Quels contenus?}

\section{$B A E Y E N$ André coord., L'élaboration des programmes de langues vivantes} dans le deuxième cycle de l'enseignement secondaire général: 15-19 ans. Rapport de l'atelier 1B, Han-sur-Lesse, Belgique, 21-26 mars 1993, Strasbourg, Conseil de l'Europe, 1993, 239 p., (Apprentissage des langues et citoyenneté européenne).

L'examen des différentes compétences à développer est illustré de nombreux exemples de documents de travail en classe.

BINES Hazel, "Curriculum Change: the Case of Special Education", British Journal of Sociology of Education, mars 1993, vol. 14, $n^{\circ} 1$, p. 75-90.

La redéfinition de l'enseignement spécial au cours de la dernière décennie en Grande-Bretagne a concerné ses différents aspects. La récente législation sur le curriculum et les changements qu'elle implique ont des implications dans ce secteur non-négligeable du système éducatif.

COSTE Daniel coord., LEHMANN Denis coord., "Langues et curriculum: contenus, programmes et parcours", Études de linguistique appliquée, juin 1995, $n^{\circ}$ 98, p. 3-127.

La première partie de ce numéro spécial retrace l'historique de la construction progressive de programme, du syllabus à la notion canadienne de curriculum multidimensionnel. Les auteurs articulent ensuite la réflexion menée par les sciences de l'éducation avec celle propre à la didactique des langues, avant de consacrer la troisième partie aux questions de continuité entre programmes, à l'apprentissage de plusieurs langues et à la formation continue.

FERRY Luc, FINKIELKRAUT Alain, LEGRAND Louis et al., "Qu'apprendre au collège?", Le Débat, déc. 1995, n 87, p. 147-180.

A travers plusieurs formes de contributions, dont le texte du rapport du Conseil national des programmes et des analyses critiques, la revue lance le débat sur la réforme du contenu de l'enseignement au collège. 
FRANCE. Direction des écoles, Programmes de l'école primaire, Paris, Centre national de documentation pédagogique, 1995, 123 p., (Une école pour l'enfant, des outils pour les maîtres).

Ce document de référence rassemble les textes officiels des horaires et programmes qui entrent en vigueur à l'école primaire à la rentrée 1995, ainsi que la liste des compétences à acquérir à chaque niveau. Il aidera les équipes pédagogiques à penser leur enseignement en fonction des trois cycles pluriannuels qui organisent la scolarité de la maternelle à la fin de l'école élémentaire.

FRANCE. Direction des Lycées et Collèges, Consultation des professeurs sur les projets de programme des voies générale et technologique des lycées: note à l'attention de la section des professeurs d'histoiregéographie, Paris, Ministère de l'Éducation nationale, oct. 1994, pag. multiple.

Cette note entre dans le cadre de l'application de la décision 136 du Nouveau contrat pour l'école. Elle est accompagnée d'un exemplaire des projets de programmes d'histoire-géographie des voies générale et technologique et du texte présentant les programmes d'histoire et géographie du lycée.

FRANCE. Direction des Lycées et Collèges, Programmes de $\mathbf{6}^{\mathbf{e}} \mathbf{1 9 9 6}$, Paris, Centre national de documentation pédagogique, Savoir-livre, 1996, 191 p., (Collège).

Ce document présente le contenu des programmes applicables, à partir de la rentrée scolaire 1996, en classe de sixième dans les disciplines suivantes: français, mathématiques, histoire-géographie, éducation civique, sciences de la vie et de la terre, technologie, enseignements artistiques, langues vivantes étrangères.

\section{LEHMANN Denis, Objectifs spécifiques en langue étrangère: les} programmes en question, Paris, Hachette Fle, 1993, 223 p., (F. Références). L'ouvrage propose un état des lieux des méthodologies propres à l'enseignement d'une langue étrangère aux publics dits spécifiques. L'auteur montre qu'une réflexion sur les besoins langagiers et sur les conditions d'apprentissage est un préalable nécessaire à l'élaboration des programmes.

\section{LEVRAT René coord., Technologie. Textes de référence: rapports COPRET}

1 et 2. Annexe: technologie et sciences dans les curricula, Sèvres, CIEP, 1992, $131 \mathrm{p}$.

Ces textes des rapports de la commission permanente de réflexion sur l'enseignement de la technologie situent les enjeux de cet enseignement en France par rapport à l'ensemble du débat européen. Ils ont conduit d'une part à la rédaction des instructions officielles, d'autre part à des propositions pour le second cycle. En postface, un article analyse l'exemple de l'Angleterre.

MOSOTHWANE Modise, "The Study of Curriculum Change in Botswana with Special Reference to Primary Science: an Historical Perspective", Curriculum Studies, mars 1995, vol. 3, n 1, p. 79-89.

Les programmes scolaires évoluent en fonction des objectifs poursuivis. L'article explique l'histoire et les raisons des changements intervenus dans l'enseignement des sciences à l'école primaire au Botswana. L'introduction des sciences physiques et de l'éducation à l'environnement correspondait à la volonté de préparer le pays à l'industrialisation et de prendre en compte la protection de la nature. 
QUÉBEC. Conseil supérieur de l'éducation, Rénover le curriculum du primaire et du secondaire: avis au ministre de l'Éducation, Sainte-Foy (Québec), Conseil supérieur de l'éducation, 1994, 116 p.

Au cours de l'année 1993-1994, le Conseil a entrepris un examen général des programmes de l'enseignement scolaire, fixés pour l'essentiel entre 1977 et 1980. Dans ce document, après avoir montré les enjeux sociaux et les finalités d'un curriculum renouvelé, il présente les améliorations possibles, globalement et aux différents niveaux d'enseignement.

ROBITAILLE David F., Curiculum Frameworks for Mathematics and Science, Vancouver, Pacific Educational Press, 1993, 102 p., (TIMSS monograph).

Ce volume est le premier d'une série de monographies réalisées par l'important groupe d'étude comparative internationale "Third international mathematics and science study». Il décrit le cadre qui sera utilisé pour examiner les programmes de mathématiques et de sciences dans cinquante pays.

VAN DER WENDE Marijk, KOUWENAAR Kees, "La recherche de la qualité: la comparaison internationale des programmes d'enseignement supérieur aux fins de l'internationalisation", Gestion de l'enseignement supérieur, juillet 1994, vol. 6, $n^{\circ} 2$, p. 261-268.

L'article examine plusieurs études récentes s'efforçant de concevoir des méthodes de comparaison qualitative de l'enseignement supérieur. Il analyse les problèmes que posent les comparaisons internationales.

\section{Quels acteurs?}

ANG Chen, ENNIS Catherine D., "Content Knowledge Transformation: an examination of the Relationship between Content Knowledge and Curricula", Teaching and Teacher Education, juillet 1995, vol. 11, $n^{\circ} 4$, p. 389-401. Les contenus de connaissances sont l'objet de transformations de la part de l'enseignant afin de les adapter aux nécessités pédagogiques. Cette étude examine comment se fait le processus de transformation en éducation physique, dans l'enseignement secondaire, aux États-Unis.

BECHER Tony ed., "Governments and the Higher Education Curriculum", European Journal of Education, sept. 1994, vol. 29, $n^{\circ} 3$, p. 227-322.

L'interaction entre gouvernement - ou Êtat - et enseignement supérieur concerne non seulement les structures, mais aussi les programmes. Les différents articles de ce numéro spécial présentent les causes, fonctions et résultats de cette implication dans des pays aussi divers que la Grande-Bretagne, l'Australie, l'Afrique du Sud, les Pays-Bas, la Roumanie, la Grèce et la Norvège.

CHITTY Clyde ed., LAWN Martin ed., "Redefining the Teacher and the Curriculum: Special Issue", Educational Review, juin 1995, vol. 47, $n^{\circ} 2$, p. 139-217.

Ce numéro spécial est consacré aux relations actuelles entre enseignants et curriculum dans plusieurs nations: Nouvelle-Zélande, États-Unis, Portugal, Suède, Australie, Pays-Bas, Écosse. Leur rôle et leur influence dans le domaine des programmes sont plus ou moins importants et se manifestent différemment selon les pays. 
FIALAIRE Jacques, "Le processus de réforme de l'enseignement en GrandeBretagne depuis 1988 ", Savoir éducation formation, sept. 1995, n³, p. 467484.

Les mesures prises ont deux objectifs principaux: assurer la mise en œuvre et l'efficacité du National Curriculum; mettre en place, en ce qui concerne la gestion des écoles, des instruments de pilotage faisant intervenir davantage le gouvernement et les parents d'élèves.

FRANCE. Conseil national des programmes, "Charte des programmes", Bulletin officiel de l'Éducation nationale, 20 fév. 1992, $n^{\circ} 8, p$. 487-492. Rédigée par le Conseil national des programmes après concertation avec les parties concernées, elle définit les procédures et le cadre d'élaboration des programmes.

JENNINGS Zellynne, "Curriculum Change in School Systems in the Commonwealth Carribbean: some Implications for the Management of Curriculum Development", International Journal of Educational Development, avril 1993, vol. 13, $n^{\circ} 2$, p. 131-143.

Après avoir situé le contexte de l'enseignement primaire et secondaire dans cette partie des Caraïbes, l'auteur examine par qui et comment se prennent les décisions de changement du curriculum. Il aborde ensuite les implications, au niveau de la gestion et de l'enseignement, de la mise en œuvre du curriculum.

\section{LEWY Arieh, L'élaboration des programmes scolaires à l'échelon central} et à l'échelon des écoles, Paris, Institut international de planification de l'éducation, 1992, 131 p., (Principes de la planification de l'éducation, 40).

Les programmes doivent-ils être conçus à l'échelon national, par l'établissement scolaire ou en associant les deux méthodes? Le document résume l'expérience des vingt dernières années et le rôle que jouent les centres d'élaboration des programmes. Il donne un aperçu historique des méthodes employées dans les différents pays du monde, montre les changements intervenus depuis la naissance du Mouvement pour la réforme des programmes scolaires (SBCD), dont il évalue l'action, et définit les termes de base utilisés.

TYREE Alexander K., "Examining the Evidence: Have States Reduced Local Control of Curriculum?», Educational Evaluation and Policy Analysis, 1993, vol. 15, $n^{\circ} 1$, p. 34-50.

L'article examine, en ce qui concerne les programmes d'enseignement, les. politiques de quatre États des États-Unis à la lumière de leur possibilité de contrôler les pratiques locales. Les résultats montrent que les politiques étatiques ont parfois réduit mais non éliminé le rôle des échelons locaux. 